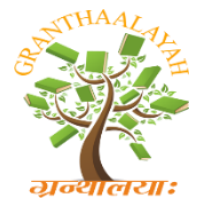

\author{
INTERNATIONAL JOURNAL OF RF
GRANTHAALAYAH \\ A knowledge Repository
}

Science

\title{
METABOLIC SYNDROME AMONG WOMEN USING HORMONAL CONTRACEPTIVES IN HARAR TOWN, EASTERN ETHIOPIA
}

\author{
Waqtola Cheneke ${ }^{* 1}$, Berhanu Sufa ${ }^{2}$ \\ ${ }^{* 1}$ Jimma University, Institute of Health, Faculty of Health Sciences, School of Medical \\ Laboratory Sciences, Ethiopia \\ ${ }^{2}$ Department of Medical Laboratory Science, Harar Health Science College, Harar, East Ethiopia
}

\begin{abstract}
Background: Metabolic syndrome is associated with the risk of developing cardiovascular disease and type 2 diabetes. Cardiovascular disease is among the most cause of death in noncommunicable diseases. The use of contraceptive is incriminated to increase the risk of cardiovascular diseases and metabolic syndrome. However, there is no clear evidence and metabolic syndrome among contraceptive users is not well described. This study is therefore focused to determine the prevalence of metabolic syndrome among contraceptive users in Harar.

Methods: A facility based cross sectional study was conducted from April - June 2014 to determine the prevalence of metabolic syndrome and associated factors in Harar town. 365 hormonal contraceptive users from three health centers and one hospital were consecutively included in the study. Socio-demographic data and anthropometric measurements were obtained. Venous blood was drawn from each study subjects and lipid profile and fasting serum glucose were measured photometrically. Prevalence of metabolic syndrome was determined using descriptive statistics and logistic regression analysis with $95 \%$ CI using statistical package for social science (SPSS, version 20 for windows).

Result: The overall prevalence of metabolic syndrome was found to be $27.7 \%$. Metabolic syndrome was found about 9 times more likely among women with age $\geq 40$ years than those with age <20 years (Odds Ratio (OR) 8.571; 95\% CI 1.004, 73.210, p = 0.050); about 2 times more likely among those with income $>1,500$ Ethiopian birr (ETB) than those with those $<1,500$ ETB (OR 1.637, 95\% CI 1.032-2.599, $\mathrm{p}=0.036$ ) and about 6 times more likely among those who used hormonal contraceptive for more than 42 weeks than those who used it for 6-17.9 weeks (OR $5.897,95 \%$ CI 2.924-11.891, $\mathrm{p}=0.000$ )

Conclusion: This study revealed that metabolic syndrome is high among contraceptive users and higher than the rate of metabolic syndrome among community and other healthy working population. Contraceptive users with older age, with higher income and those who used the drug for longer time were found to have more metabolic syndrome than their counter parts.
\end{abstract}

Keywords: Prevalence; Metabolic Syndrome; Hormonal Contraceptives; Risk Factor; Ethiopia.

Cite This Article: Waqtola Cheneke, and Berhanu Sufa. (2019). "METABOLIC SYNDROME AMONG WOMEN USING HORMONAL CONTRACEPTIVES IN HARAR TOWN, 
EASTERN ETHIOPIA.” International Journal of Research - Granthaalayah, 7(10), 400-406. https://doi.org/10.29121/granthaalayah.v7.i10.2019.414.

\section{Introduction}

Non-communicable diseases are becoming major public health concern even in developing countries like Ethiopia where communicable diseases were being given major attention than the non-communicable. Morbidity and mortality due to these non-communicable diseases are extremely increasing. Currently, in the world, the most leading cause of death is cardiovascular disease which is non-communicable. ${ }^{[1,2]}$

Metabolic syndrome which is a condition comprised from a group of at least 3 of the following 5 medical conditions: central obesity, high blood pressure, high blood sugar, high serum triglycerides, and low serum high-density lipoprotein (HDL) is associated with the risk of developing cardiovascular disease and type 2 diabetes. ${ }^{[3,4]}$

Metabolic syndrome is defined differently by different agencies such as International Diabetes Federation (IDF), World Health Organization (WHO), European Group for the study of Insulin Resistance (EGIR), the US National Cholesterol Education Adult Treatment Panel III (NCEP), and American Heart Association (AHA). For the sake of this study we chose the NCEP definition which requires at least 3 of the 5 medical conditions indicated above.

Although the cause of this syndrome is an area of ongoing medical research, its occurrence among different types of population and predisposing factors are being communicated to the scientific world. For example, in the US about a quarter of the adult population has metabolic syndrome, and the prevalence increases with age, with racial and ethnic minorities being particularly affected. $[5,6]$

In Ethiopia, few studies have been published showing the prevalence of metabolic syndrome among hospital outpatients (26\%), PLWHA (23.5\%), psychiatric patients (28.9\%), type 2 diabetic patients $(45.9 \%)$, and healthy working adults $(12.5 \%){ }^{[7-11]}$

Hormonal contraceptive use is also claimed to affect cardiac function, blood pressure, fat and carbohydrate metabolism. In the presence of other cardiovascular risk factors, the combined oral contraceptive (COC) can enhance the risk of vascular disease. ${ }^{[12]}$ Prolonged use of hormonal contraceptives by females during their reproductive age can induce metabolic changes that may contribute to an increased risk of coronary heart. ${ }^{[13]}$ However, there is limited study that could describe the prevalence of metabolic syndrome among contraceptive users.

This study is therefore designed to assess the prevalence of metabolic syndrome among contraceptive users in Harar Ethiopia. 


\section{Materials and Methods}

\subsection{Study setting and study participants}

A cross sectional study was conducted from April to June, 2014 in Harar Eastern Ethiopia, which is $515 \mathrm{~km}$ away from Addis Ababa. A total of 365 hormonal contraceptive users having complete recorded data, satisfied the inclusion criteria and volunteer to participate in the study were included consecutively from 3 health centers and 1 hospital in the town.

\subsection{Data Collection and Laboratory Testing}

Socio demographic and clinical data were collected using structured questionnaire and checklist. Overnight fasting morning blood specimen of about $10 \mathrm{ml}$ of venous blood was collected from each study participants into clean serum separator test tubes. Serum was separated from the blood sample and then blood glucose level, total cholesterol, triglycerides and LDL-C was determined using Mindray BS-120 chemistry auto-analyzer machine (Shenzhen Mindray Bio-Medical Electronics CO., LTD.). HDL-C was calculated in serum specimens having a TG value $<400 \mathrm{mg} / \mathrm{dl}$ by reverse method using Friedewald formula.

Standard operating procedures and manufacturer instructions were strictly followed throughout the procedures and all reagents were prepared according to the manufacturer's instruction. Quality control run was undertaken for all laboratory tests in this study.

\subsection{Statistical Analysis}

Data was edited, cleaned, coded and entered using statistical software (Epidata, version 3.1) and then exported to and analyzed with SPSS, version 20 for windows. Mean, standard deviation and frequency of Quantitative variables were calculated. Bivariate and multivariate logistic regression analysis was calculated with $95 \% \mathrm{CI}$ to evaluate the possible association of the variables and Pvalue of less than 0.05 was considered as statistically significant.

\subsection{Ethical Consideration}

Ethical clearance was obtained from Jimma university ethical review committee and official letter was written to Harari Regional State Health Bureau. For voluntary participation, research subjects' signed for informed consent based on explicit information of any possible risk, harm and even discomfort caused by data/sample collection procedures as well as any benefits. Moreover, individuals who were found to have abnormal results including dyslipidemia and metabolic syndrome were sent to the health care workers for further management of the problem.

\section{Results}

\subsection{Demographic and Anthropometric Data}

A total of 365 consented women who came for their family planning service follow up and used hormonal contraceptives for more than six months were enrolled in this study with overall response rate of $100 \%$. 
The mean $( \pm \mathrm{SD})$ age of the study participants was $30.1( \pm 6.7)$ years. Of the total participants, $13(3.6 \%)$ were below 20 years old, $163(44.7 \%)$ were $20-29$ years old, 153(41.9\%) were 30-39 and $36(9.9 \%)$ were $40-49$ years of age.

The mean height and weight of the study participants were $161( \pm 0.06) \mathrm{cm}$ and $57.9( \pm 6.8) \mathrm{kg}$, respectively. By religion, 187(51.2\%) of the study subjects were Muslim, 126(34.5\%) were orthodox and the remaining 52(14.2\%) were protestant. Ethnically, 162 (44.4\%), 95(26.0\%), 73(20\%), and 35(9.6\%) were Oromo, Amhara, Harari and other ethnic groups, respectively. Educationally, 206(56.4\%), 81(21.2\%), and 78(21.4\%) attended above secondary school, were primary and junior grades, and can't read and write, respectively. Occupationally, 157 (43\%), 138 (37.8\%), $67(18.4 \%)$ and $2(0.5 \%)$ were housewives, employed, merchant or self-employed and students, respectively. Three hundred and seven $(84.1 \%)$ were married. The mean monthly income of the family was $1,786.6$ ETB.

\subsection{Clinical and Laboratory Data in The Study Participants}

The mean $( \pm \mathrm{SD})$ levels of TC, LDL-C, HDL-C, the TC/HDL-C ratio, and TG were $186( \pm 27) \mathrm{mg} / \mathrm{dl}$, $121( \pm 31) \mathrm{mg} / \mathrm{dl}, 45.21( \pm 7.7) \mathrm{mg} / \mathrm{dl}, 4.44$, and $108( \pm 3.45) \mathrm{mg} / \mathrm{dl}$, respectively. Out of 365 study subjects only $12(3.3 \%)$ had a systolic blood pressure greater than $140 \mathrm{mmHg}$ and $23(6.3 \%)$ of the study subjects had a diastolic blood pressure greater than $90 \mathrm{mmHg}$. The mean $( \pm \mathrm{SD})$ systolic and diastolic blood pressure were $120 \mathrm{mmHg}( \pm 9.5 \mathrm{mmHg})$ and $78 \mathrm{mmHg}( \pm 6.8 \mathrm{mmHg})$, respectively. Thirty $(8.2 \%)$ of the study subjects had a fasting blood sugar greater than $126 \mathrm{mg} / \mathrm{dl}$ and $73(20 \%)$ of them had BMI $>25 \mathrm{~kg} / \mathrm{m}^{2}$ and only $9(2.5 \%)$ of them had a BMI $<18.5 \mathrm{~kg} / \mathrm{m}^{2}$. The mean $( \pm \mathrm{SD})$ BMI among the study participants was $22.52\left( \pm 2.36 \mathrm{~kg} / \mathrm{m}^{2}\right)$.

Of the total study subjects, $94(25.8 \%)$ had a waist to hip ratio $>0.85$. Additionally, $16(4.4 \%)$ had a family history of hypertension; $21(5.8 \%)$ had a family history of diabetes mellitus and 11(3\%) has a family history of obesity. Of the total study participants 203(55.6\%) drink coffee; $163(44.7 \%)$ of them drink at least once a day and $40(11 \%)$ of them drink more than twice a day. Of the total study subjects $186(51 \%)$ make a physical exercise for at least 30 minutes a day with $176(48.2 \%)$ exercise less than three times a week and $10(2.7 \%)$ exercise greater than 4 times a week. Sixteen $(4.4 \%)$ drink alcohol at least once a week and $69(18.9 \%)$ of the study subjects chew khat at least once a week.

Metabolic syndrome of overall prevalence of $27.7 \%$ was found (Fig 1). The presence of metabolic syndrome was compared with variables of the study subjects including: age, occupation, income, religion, marital status, educational status, type of hormonal contraceptive used, duration of hormonal contraceptive use and etc. The presence of metabolic syndrome was found to be statistically significantly different among age categories, income and duration of hormonal contraceptive use.

Metabolic syndrome was found about 9 times more likely among women with age $\geq 40$ years than those with age <20 years (Odds Ratio (OR) 8.571; 95\% CI 1.004, 73.210, p = 0.050); about 2 times more likely among those with income $>1,500$ Ethiopian birr (ETB) than those with those $<1,500$ ETB (OR 1.637, 95\% CI 1.032-2.599, $\mathrm{p}=0.036)$ and about 6 times more likely among those who 
used hormonal contraceptive for more than 42 weeks than those who used it for 6-17.9 weeks (OR 5.897, 95\% CI 2.924-11.891, p=0.000) (Table 1).

\section{Discussion}

Metabolic syndrome is a common public health concern, the prevalence of which is rising steadily. In this study the overall prevalence of metabolic syndrome among women using hormonal contraceptives was found to be $27.7 \%$. In the study area it was fond that metabolic syndrome were statistically different among different age categories, different income range and different duration of hormonal contraceptive use.

The finding of this current study, metabolic syndrome of contraceptive users in Harar, (27.7\%), is similar with the study finding from Jimma among OPD patients $(26 \%)^{[7]}$; among PLWHIV $(23.5 \%)^{[8]}$; among psychiatric patients $(28.9 \%)^{[9]}$ and among T2DM in Hawasa $(33.8 \%) .{ }^{[10]}$ This might indicate that the use of hormonal contraceptive have not this much effect in increasing the metabolic syndrome rate. This could be clarified by undertaking another large study by comparing contraceptive use with those without its use.

On the other hand, the finding from current study have more percentage of metabolic syndrome as compared with a study from a community based in Mizan-Aman town $(9.6 \%)^{[14]}$ and a study from apparently healthy working adults in Addis Ababa (12.5\%). ${ }^{[1]}$ This could be explained as that contraceptive users in Harar might not controlled their life styles to control their blood lipid levels. A study on the contraceptive users of the same population from Harar ${ }^{[19]}$ identified that there is high rate of dyslipidemia and emphasized the urgent need for prevention, early detection and treatment of dyslipidemia. Additionally, the contraceptive drug use could have effect to increase the metabolic syndrome rate than those without the drug use.

Moreover, the metabolic syndrome status of these contraceptive users in Harar is less than the finding from a study in University of Gondar hospital among T2DM $(66.7 \% \text { and } 70.3 \%)^{[15,16]}$; hypertensive patients $(40.7 \%)^{[17]}$ and from Hawasa university comprehensive hospital among hypertensive patients $(48.7 \%)^{[18]}$. This difference might arise from the fact that both the diabetic patients and hypertensive patients had not controlled their disease condition status. This is because the parameters used to define metabolic syndrome are high blood glucose, dyslipidemia and high blood pressure.

Metabolic syndrome in these contraceptive users is statistically significantly different among age groups, different income level and duration of contraceptive use. This means study participants with older age of greater than 40 years, income status of greater than 1500 birr and duration of contraceptive drug use of more than 42 weeks were more prone to have metabolic syndrome than their counter parts. In similar fashion a study from Hawasa university hospital among hypertensive patients had more metabolic syndrome rate among those with income greater than 1200 birr $^{[18]}$. Similarly, studies from Jimma ${ }^{[9]}$, Hawasa ${ }^{[10]}$ and Gondar ${ }^{[15]}$ had showed that participants with older age have more metabolic syndrome than their counter parts. 


\section{Conclusion and Recommendations}

This study revealed that metabolic syndrome is high among contraceptive users and higher than the rate of metabolic syndrome among community and other healthy working population. Contraceptive users with older age, with higher income and those who used the drug for longer time were found to have more metabolic syndrome than their counter parts.

The results of this study emphasize the urgent need for a public health strategy for the prevention, detection, and treatment of metabolic syndrome in Harar town. Public health measures should continue to emphasize the importance of healthy feeding habits and screening for different metabolic disorders as well as provide and monitor the quality of family planning services aiming to obtain a better quality of life. Moreover, large scale longitudinal study is needed to determine the effect of hormonal contraceptives on metabolic syndrome. Thus, this study can be used as baseline information for further studies related to effect of hormonal contraceptives on metabolic syndrome level.

\section{Acknowledgments}

We would like to thank our data collectors. Our appreciation also goes to the participants of the study who willingly participated in the study to give all the necessary information for the study.

\section{References}

[1] WHO. Cardiovascular diseases (VDs) Key facts; 17 May 2017. https://www.who.int/newsroom/fact-sheets/detail/cardiovascular-diseases-(cvds). Accessed on 20 July 2019.

[2] WHO. Noncommunicable diseases (NCD) Key facts; 1 June 2018. https://www.who.int/newsroom/fact-sheets/detail/noncommunicable-diseases. Accessed on 20 July 2019.

[3] Kaur J. A comprehensive review on metabolic syndrome [retracted in: Cardiol Res Pract. 2019 Jan 31;2019:4301528]. Cardiol Res Pract. 2014;2014:943162. doi:10.1155/2014/943162

[4] Felizola, Saulo JA. Ursolic acid in experimental models and human subjects: Potential as an antiobesity/overweight treatment?. 2015; doi:10.13140/RG.2.1.4502.4804

[5] Falkner B, Cossrow ND. Prevalence of metabolic syndrome and obesity-associated hypertension in the racial ethnic minorities of the United States. Current Hypertension Reports. 2014; 16 (7): 449. doi:10.1007/s11906-014-0449-5. PMC 4083846. PMID 24819559

[6] Beltrán-Sánchez H, Harhay MO, Harhay MM, McElligott S. Prevalence and trends of metabolic syndrome in the adult U.S. population, 1999-2010. Journal of the American College of Cardiology. 2013; 62 (8): 697 703. doi:10.1016/j.jacc.2013.05.064. PMC 3756561. PMID 23810877

[7] Abda E, Hamza L, Tessema F, Cheneke W. Metabolic syndrome and associated factors among outpatients of Jimma University Teaching Hospital. Diabetes Metab Syndr Obes. 2016;9:47-53. Published 2016 Mar 4. doi:10.2147/DMSO.S97561

[8] Bosho DD, Dube L, Mega TA, Adare DA, Tesfaye MG, Eshetie TC. Prevalence and predictors of metabolic syndrome among people living with human immunodeficiency virus (PLWHIV). Diabetol Metab Syndr. 2018;10:10. Published 2018 Feb 21. doi:10.1186/s13098-0180312-y

[9] Asaye S, Bekele S, Tolessa D, Cheneke W. Metabolic syndrome and associated factors among psychiatric patients in Jimma University Specialized Hospital, South West Ethiopia. Diabetes Metab Syndr. 2018 Sep;12(5):753-760. doi: 10.1016/j.dsx.2018.04.037. Epub 2018 Apr 24. 
[10] Woyesa SB, Hirigo AT, Wube TB. Hyperuricemia and metabolic syndrome in type 2 diabetes mellitus patients at Hawassa university comprehensive specialized hospital, South West Ethiopia. BMC Endocr Disord. 2017;17(1):76. Published 2017 Dec 12. doi:10.1186/s12902-0170226-y

[11] Tran, B. Gelaye, B. Girma, et al., Prevalence of Metabolic Syndrome among Working Adults in Ethiopia, International Journal of Hypertension, vol. 2011, Article ID 193719, 8 pages, 2011. https://doi.org/10.4061/2011/193719.

[12] Wiegratz I, Thaler CJ. Hormonal contraception--what kind, when, and for whom?. Dtsch Arztebl Int. 2011;108(28-29):495-506. doi:10.3238/arztebl.2011.0495

[13] Jamil S, Siddiq A. Comparison of CVD risk associated with the long term use of contraceptives in young females. J Appl Pharm Sci. 2012;2(11):062-6.

[14] Kerie S, Menberu M, Geneto M. Metabolic syndrome among residents of Mizan-Aman town, South West Ethiopia, 2017: A cross sectional study. PLoS One. 2019;14(1):e0210969. Published 2019 Jan 31. doi:10.1371/journal.pone.0210969

[15] Birarra MK, Gelayee DA. Metabolic syndrome among type 2 diabetic patients in Ethiopia: a crosssectional study. BMC Cardiovasc Disord. 2018;18(1):149. Published 2018 Jul 17. doi:10.1186/s12872-018-0880-7

[16] Biadgo B, Melak T, Ambachew S, et al. The Prevalence of Metabolic Syndrome and Its Components among Type 2 Diabetes Mellitus Patients at a Tertiary Hospital, Northwest Ethiopia. Ethiop J Health Sci. 2018;28(5):645-654. doi:10.4314/ejhs.v28i5.16

[17] Tachebele B, Abebe M, Addis Z, Mesfin N. Metabolic syndrome among hypertensive patients at University of Gondar Hospital, North West Ethiopia: a cross sectional study. BMC CardiovasC Disord. 2014;14:177. Published 2014 Dec 6. doi:10.1186/1471-2261-14-177

[18] Tadewos A, Egeno T, Amsalu A. Risk factors of metabolic syndrome among hypertensive patients at Hawassa University Comprehensive Specialized Hospital, Southern Ethiopia. BMC CardiovasC Disord. 2017;17(1):218. Published 2017 Aug 8. doi:10.1186/s12872-017-0648-5

[19] Sufa B, Abebe G, Cheneke W. Dyslipidemia and associated factors among women using hormonal contraceptives in Harar town, Eastern Ethiopia. BMC Res Notes. 2019;12(1):120. Published 2019 Mar 4. doi:10.1186/s13104-019-4148-9

\footnotetext{
*Corresponding author.

E-mail address: waqtola.cheneke@ ju.edu.et/waqtolachalt@yahoo.com
} 